Supporting information

\title{
Calcium-Assisted in-Situ Formation of Perovskite Nanocrystals for Luminescent Green and Blue Emitters
}

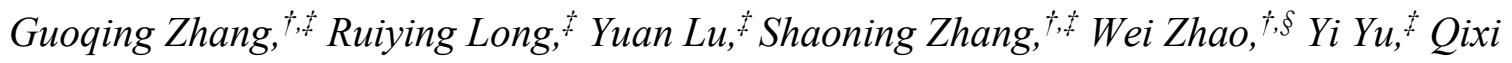
Mi, ${ }^{*}, \dot{t}$ Peng Qin, ${ }^{*}, \dot{t}, \S$ and Fuqiang Huang ${ }^{*}, \dot{t}, \xi, \perp$

$\dagger$ State Key Laboratory of High Performance Ceramics and Superfine Microstructure, Shanghai Institute of Ceramics, Chinese Academy of Sciences, Shanghai 200050, China

\$ School of Physical Science and Technology, ShanghaiTech University, Shanghai 201210, China

$\S$ Center of Materials Science and Optoelectronics Engineering, University of Chinese Academy of Science, Beijing 100049, China

$\perp$ State Key Laboratory of Rare Earth Materials Chemistry and Applications, College of Chemistry and Molecular Engineering, Peking University, Beijing 100871, China

\section{Corresponding Authors}

*E-mail: miqx@shanghaitech.edu.cn (Q.M.).

*E-mail: qinpeng@mail.sic.ac.cn (P.Q.).

*E-mail: huangfq@mail.sic.ac.cn (F.H.). 

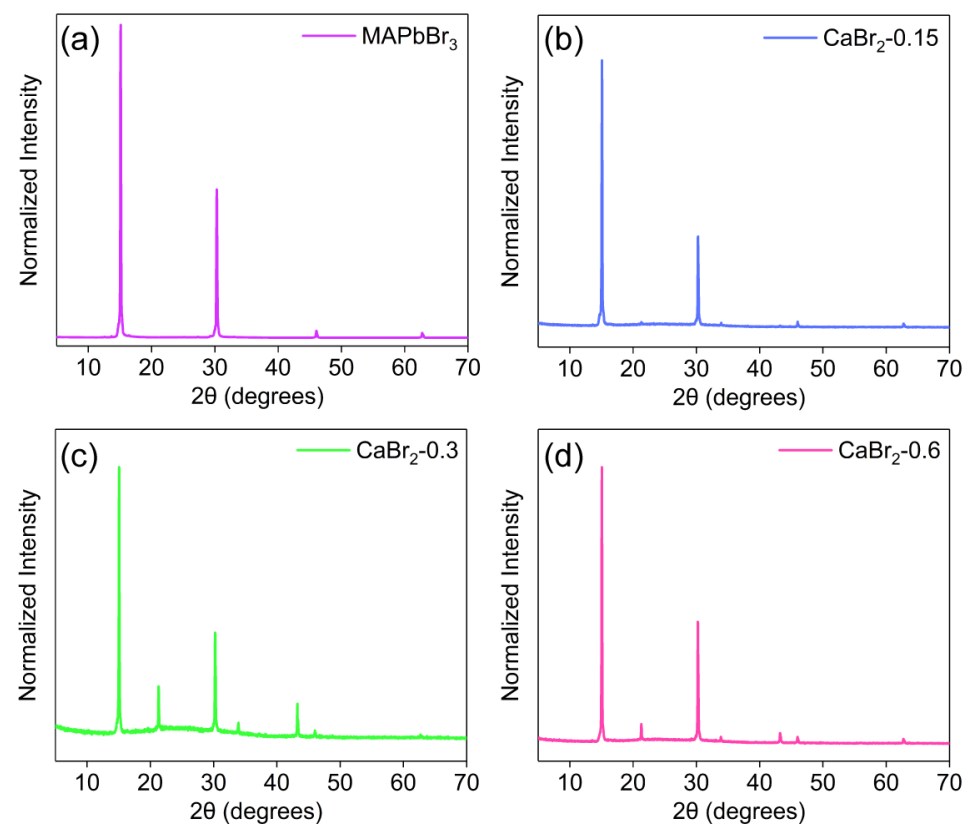

Figure S1. The normalized X-ray diffraction patterns of the (a) pristine $\mathrm{MAPbBr}_{3}$, (b) $\mathrm{CaBr}_{2}-0.15$, (c) $\mathrm{CaBr}_{2}-0.3$, and (d) $\mathrm{CaBr}_{2}-0.6$ films. 

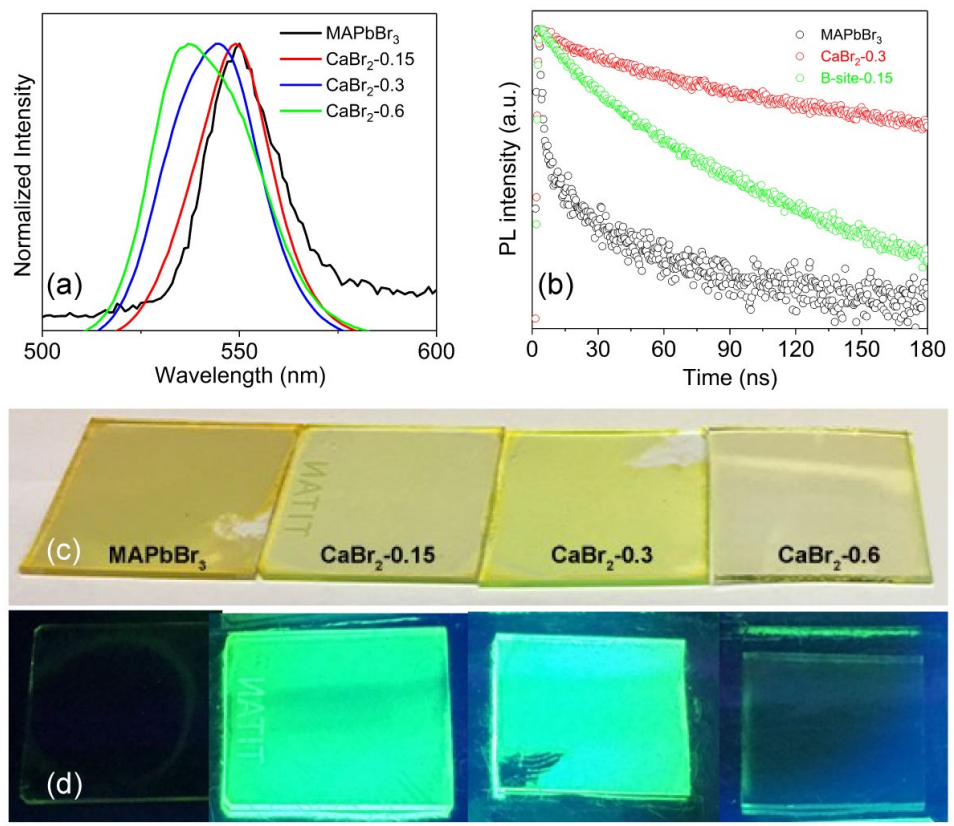

Figure S2. (a) The normalized steady-state photoluminescence (PL) spectra of $\mathrm{MAPbBr}$, $\mathrm{CaBr}_{2}-0.15, \mathrm{CaBr}_{2}-0.3$, and $\mathrm{CaBr}_{2}-0.6$ films. (b) Time-resolved PL spectra of $\mathrm{MAPbBr}_{3}$, $\mathrm{CaBr}_{2}-0.3$, and B-site-0.15.(c) optical images of $\mathrm{MAPbBr}_{3}, \mathrm{CaBr}_{2}-0.15, \mathrm{CaBr}_{2}-0.3$, and $\mathrm{CaBr}_{2}-0.6$ films. (d) $\mathrm{PL}$ image of $\mathrm{MAPbBr}_{3}, \mathrm{CaBr}_{2}-0.15, \mathrm{CaBr}_{2}-0.3$, and $\mathrm{CaBr}_{2}-0.6$ films under ultraviolet lamp excitation. 

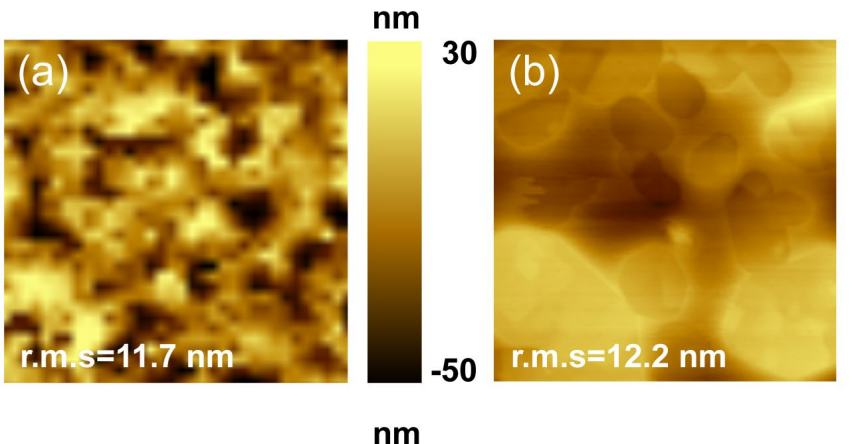

$\begin{array}{ll}\mathrm{nm} & \\ & \end{array}$
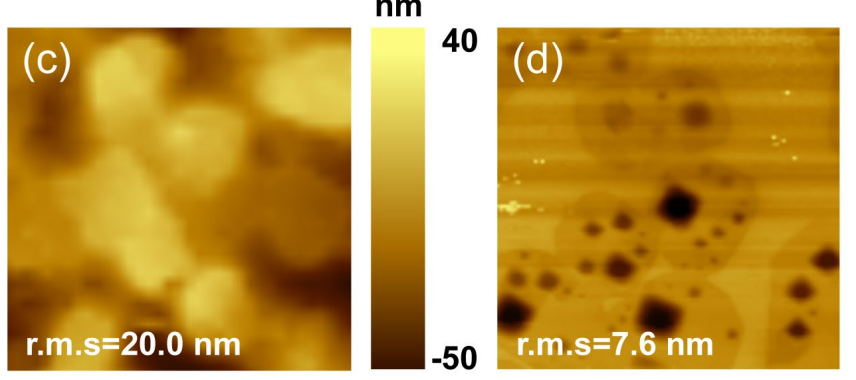

nm 20

20

Figure S3. AFM images of (a) $\mathrm{MAPbBr}_{3}$, (b) $\mathrm{CaBr}_{2}-0.15$, (c) $\mathrm{CaBr}_{2}-0.3$ and (d) $\mathrm{CaBr}_{2}-0.6$ films. The scan area of all images is $1.5 \mu \mathrm{m} \times 1.5 \mu \mathrm{m}$. 

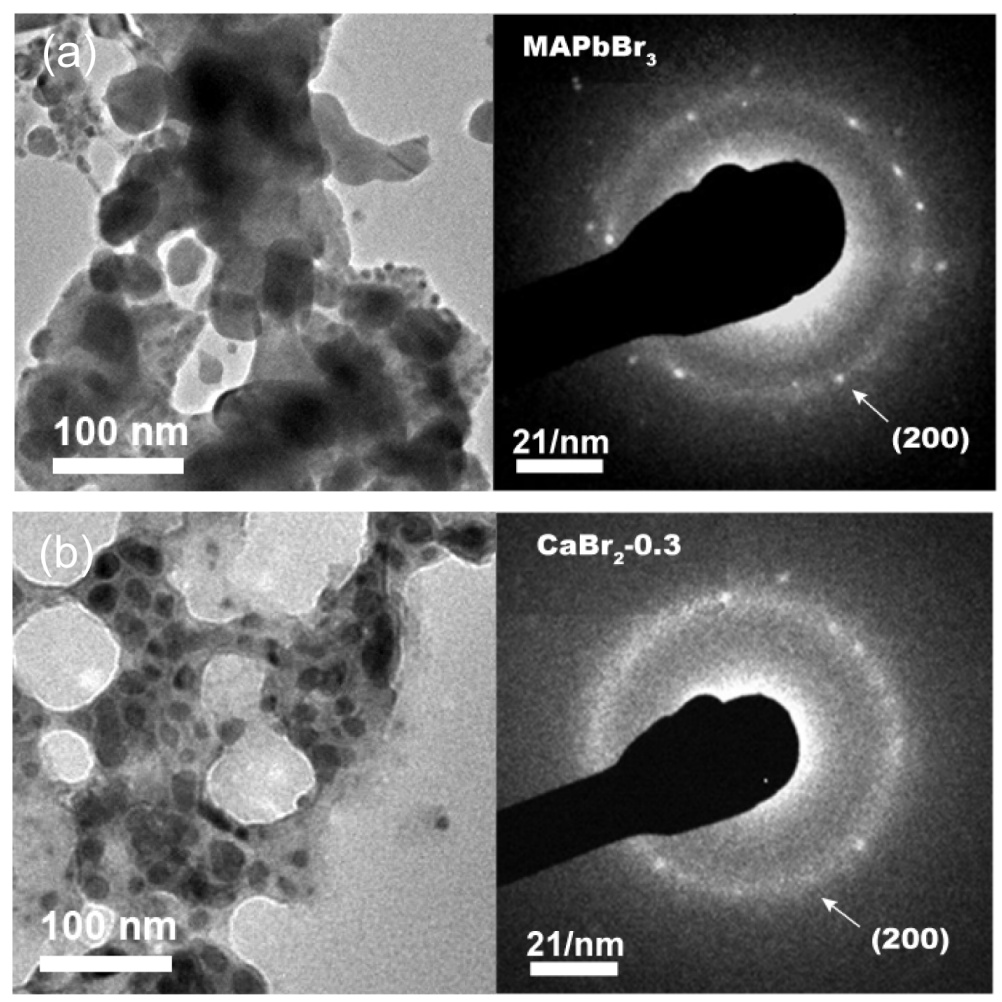

Figure S4. Transmission electron microscopic images and the corresponding SAED patterns for (a) $\mathrm{MAPbBr}_{3}$ and (b) $\mathrm{CaBr}_{2}-0.3$ films.
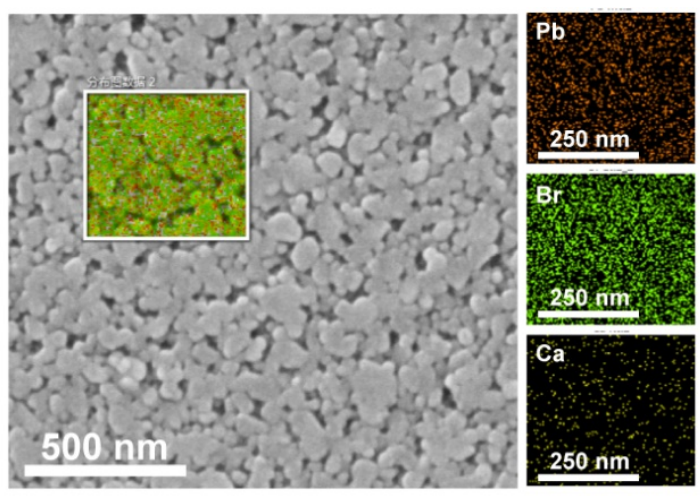

Figure S5. Scanning electron microscopic image and the corresponding elemental mapping images of the $\mathrm{CaBr}_{2}-0.3$ thin film. 

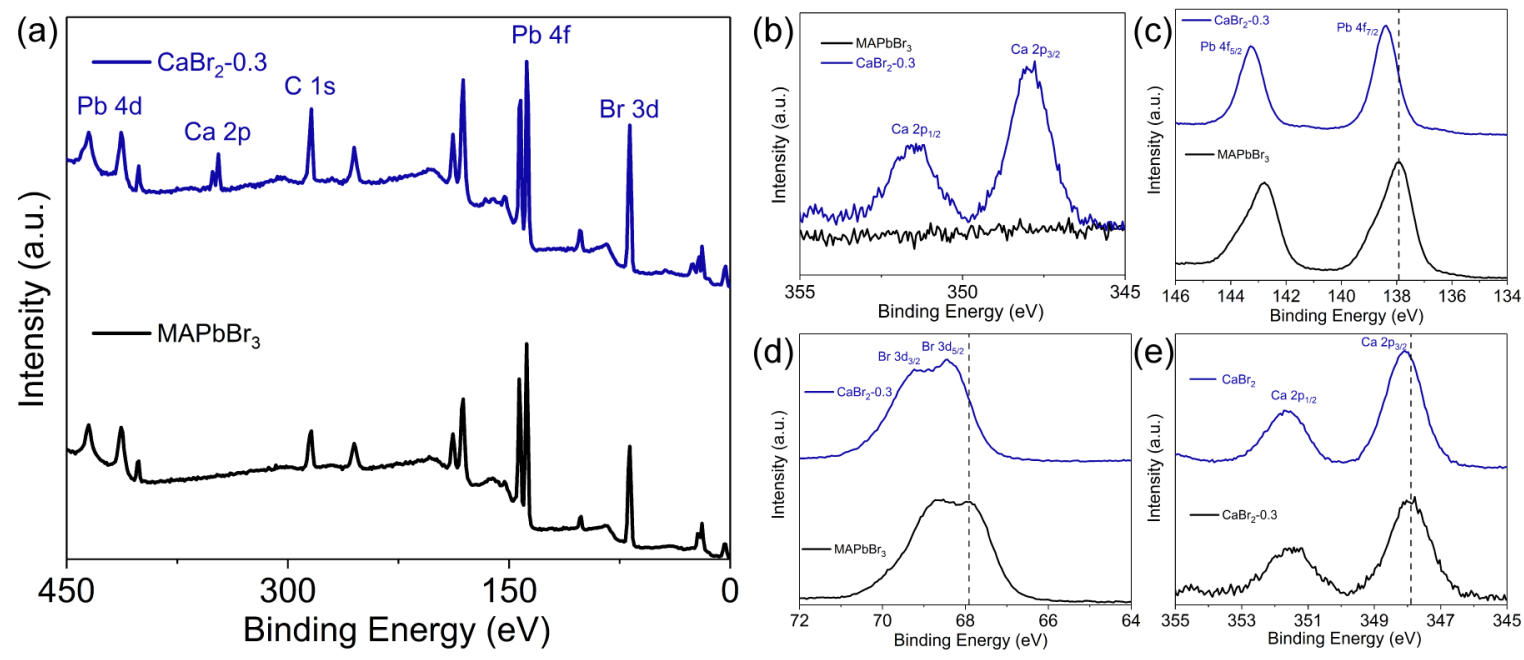

Figure S6. X-ray photoelectron spectroscopy (XPS) of pristine $\mathrm{MAPbBr}_{3}$ and $\mathrm{CaBr}_{2}-0.3$ thin films. (a) survey spectra of pristine $\mathrm{MAPbBr}_{3}$ and $\mathrm{CaBr}_{2}-0.3$ thin films. High-resolution XPS spectrum of (b) $\mathrm{Ca} 2 \mathrm{p}$, (c) $\mathrm{Pb} 4 \mathrm{f}$ and (d) $\mathrm{Br} 3 \mathrm{~d}$ for pristine $\mathrm{MAPbBr}_{3}$ and $\mathrm{CaBr}_{2}-0.3$ thin films. (e) High-resolution XPS spectrum of $\mathrm{Ca} 2 \mathrm{p}$ of $\mathrm{CaBr}_{2}-0.3$ and pure $\mathrm{CaBr}_{2}$ thin films.
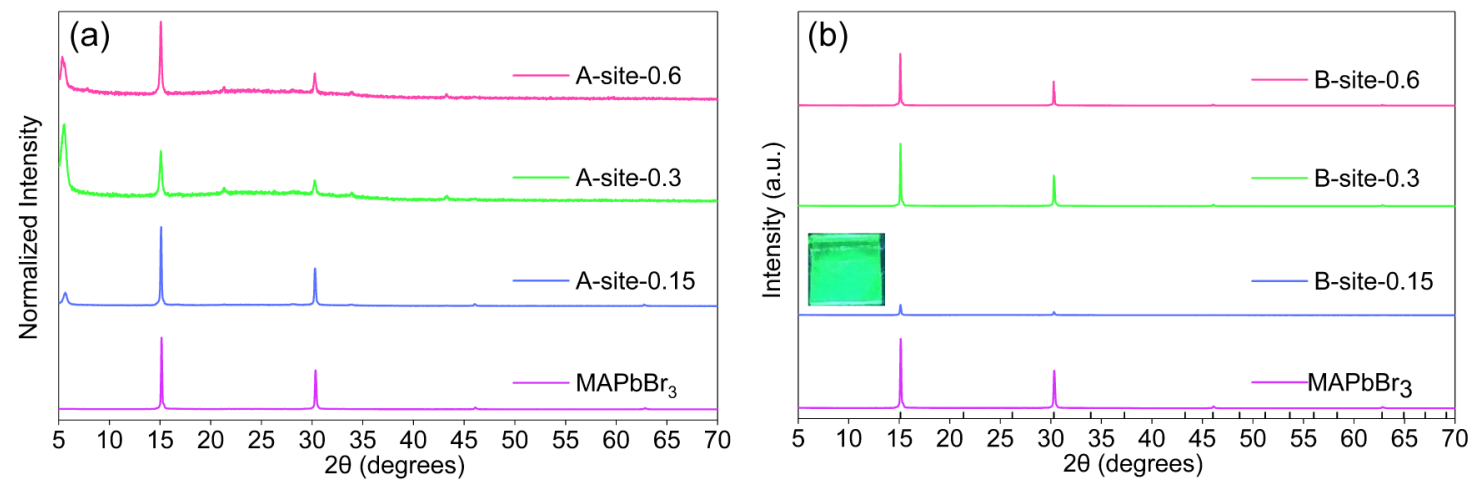

Figure S7. (a) and (b) X-ray diffraction spectra of the $\mathrm{MAPbBr}_{3}$, A-site and B-site doping films. PL image (inset) of B-site-0.15 film under ultraviolet lamp excitation. The vertical bar (black) represents the peak positions of an ideal cubic $\mathrm{MAPbBr}_{3}$ perovskite. 
(a)

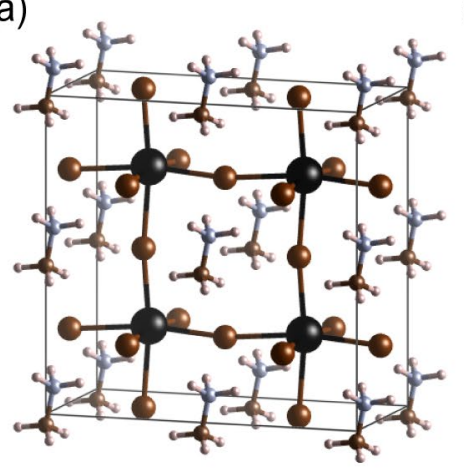

(d)

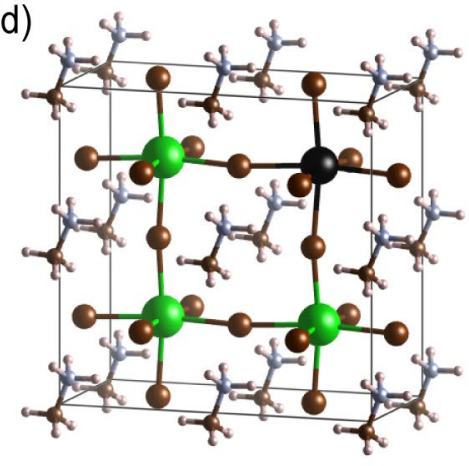

(b)

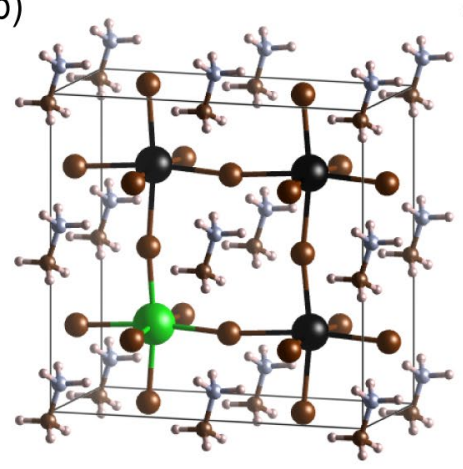

(c)

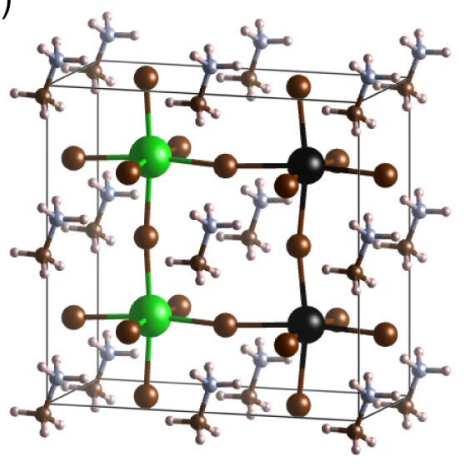

(e)

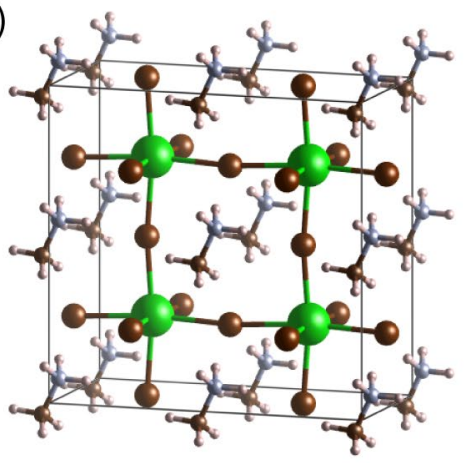

Figure S8. Optimized supercell models of five different substitution ratios $x=$ (a) 0 , (b) 0.25, (c) 0.50 , (d) 0.75 and (e) 1 , in $\mathrm{MAPb}_{1-x} \mathrm{Ca}_{x} \mathrm{Br}_{3}$. Black balls stand for $\mathrm{Pb}^{2+}$ and green ones for $\mathrm{Ca}^{2+}$. 

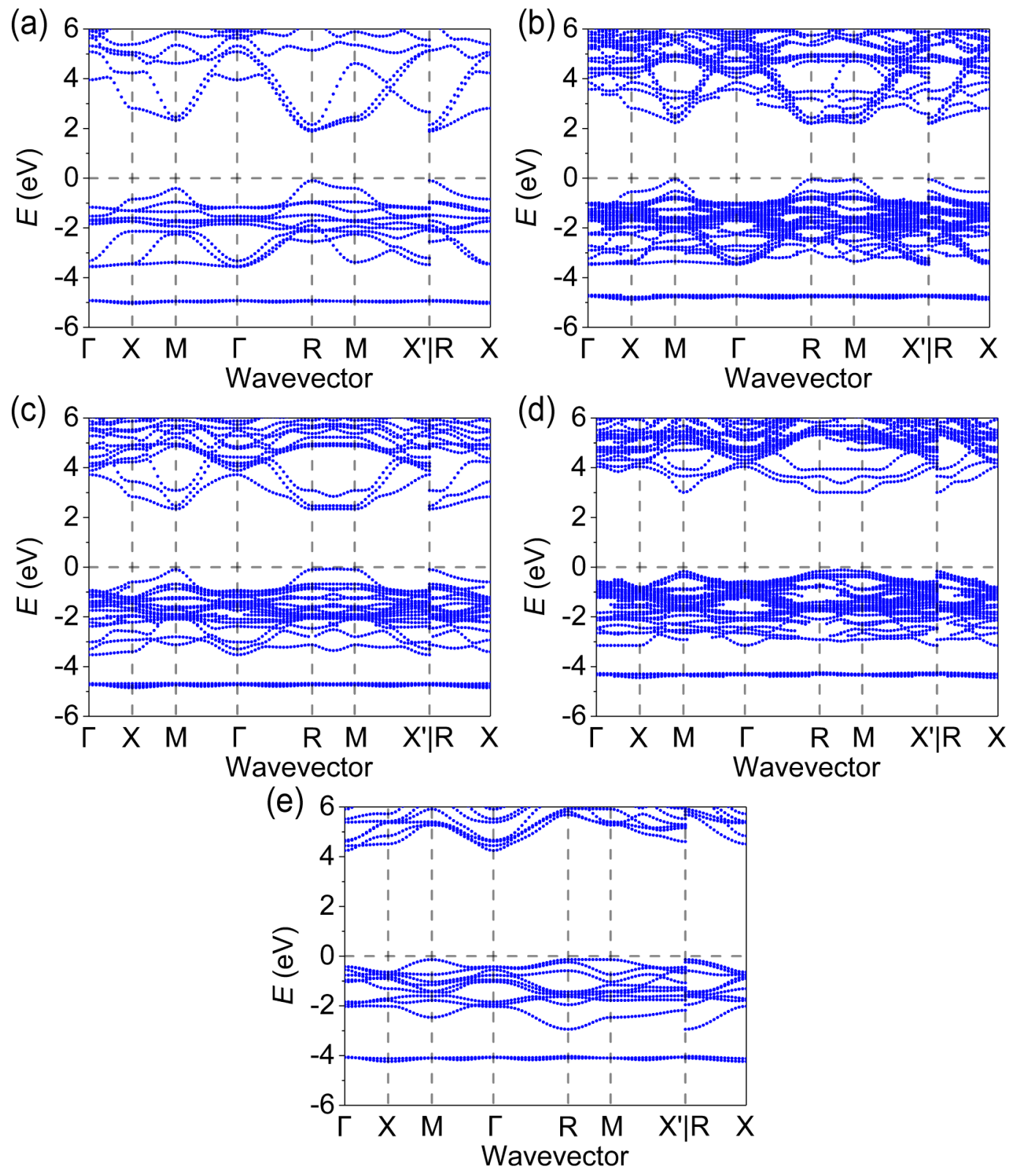

Figure S9. Unfolded electronic band structures of different substitution ratios $x=$ (a) 0 , (b) 0.25 , (c) 0.50 , (d) 0.75 and (e) 1, in $\mathrm{MAPb}_{1-x} \mathrm{Ca}_{x} \mathrm{Br}_{3}$. 

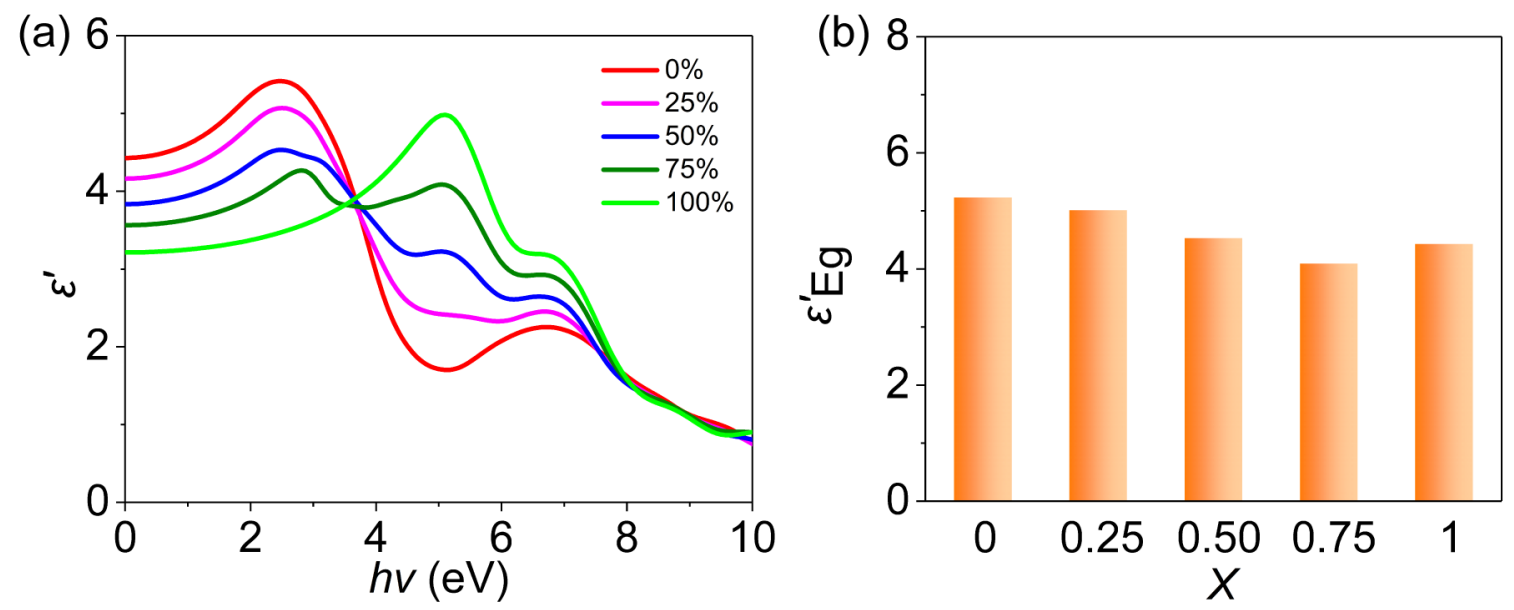

Figure S10. Calculated dielectric constant $\varepsilon^{\prime}$ of $\mathrm{MAPb}_{1-x} \mathrm{Ca}_{x} \mathrm{Br}_{3}$. (a) $\varepsilon^{\prime}$ as a function of photon energy. (b) $\varepsilon^{\prime}$ near the absorption edge of $\mathrm{MAPb}_{1-x} \mathrm{Ca}_{x} \mathrm{Br}_{3}$ at mixing ratios.

(a)
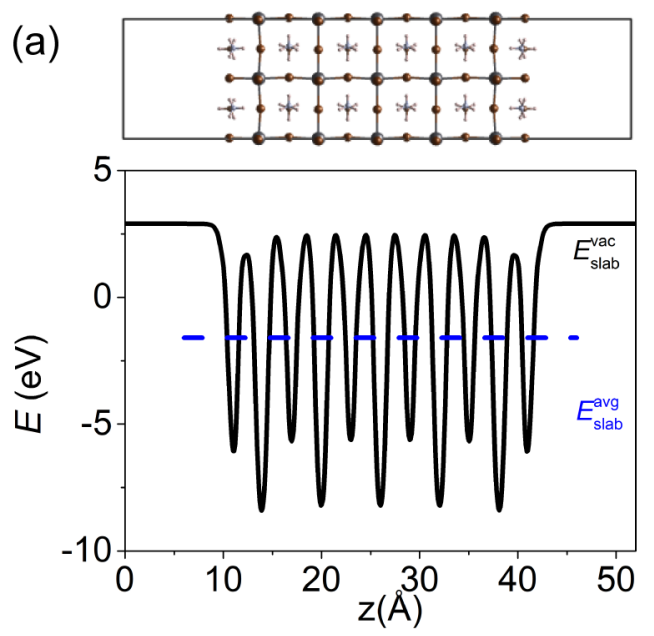

(b)
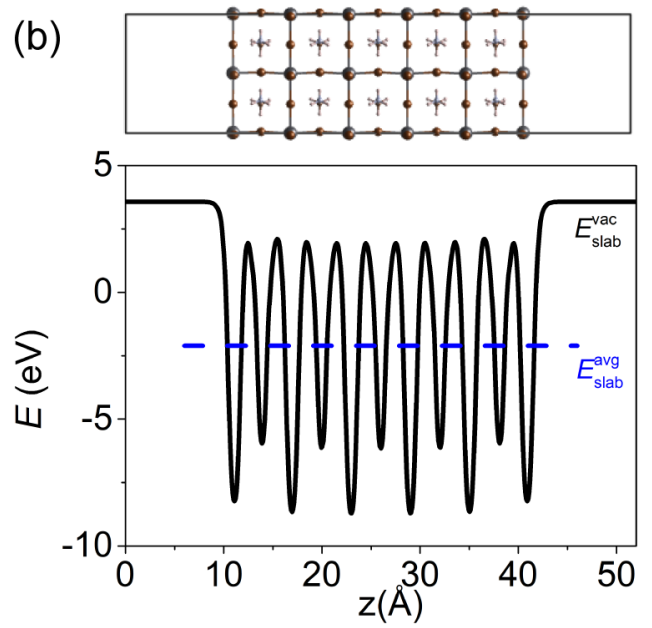

Figure S11. Relaxed symmetric slab models of the $\mathrm{MAPbBr}_{3}(001)$ surface with (a) MA and (b) $\mathrm{PbBr}_{2}$ termination and the corresponding electrostatic potential along $z$ direction. In each case, the blue dash lines indicate the macroscopic average $E_{\text {slab }}^{\mathrm{avg}}$ of the electrostatic potentials. 

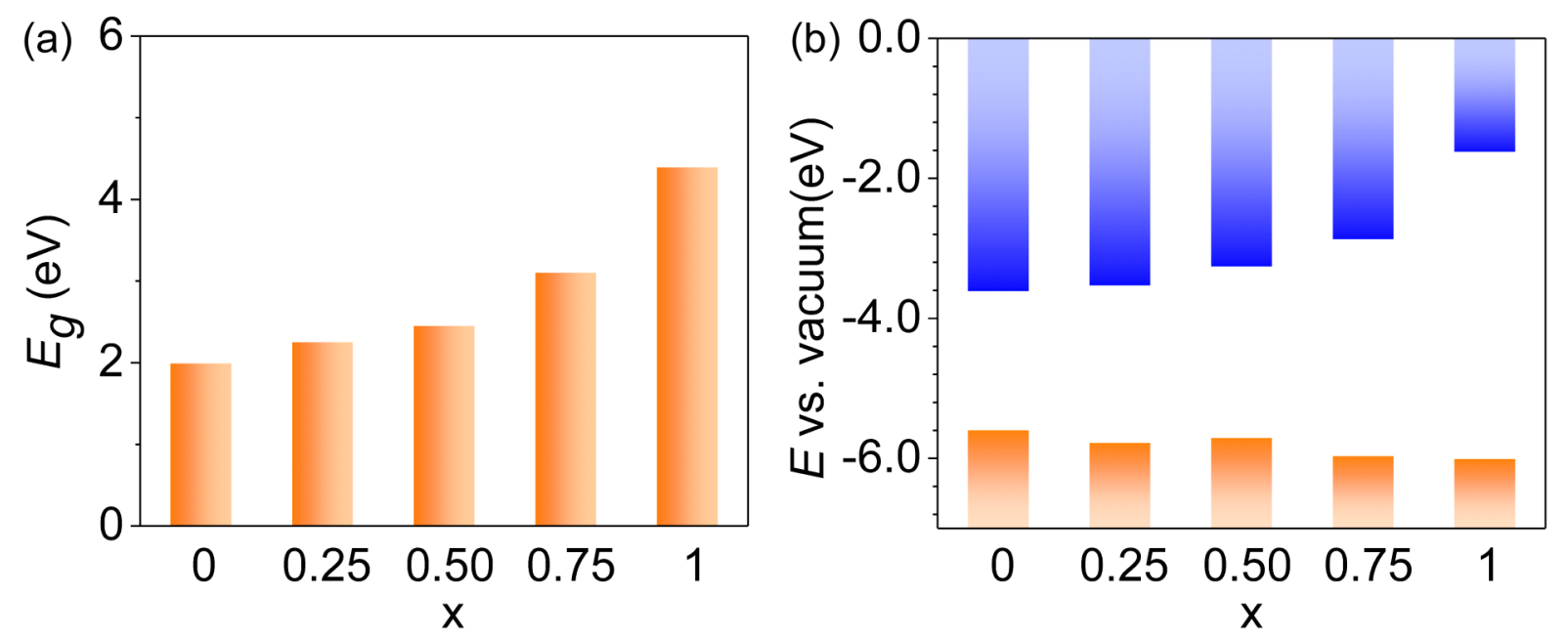

Figure S12. Comparisons of $\mathrm{MAPb}_{1-x} \mathrm{Ca}_{x} \mathrm{Br}_{3}$ in (a) bandgap energy, (b) absolute energy level of bandedges. Detailed values are listed in Table S6.
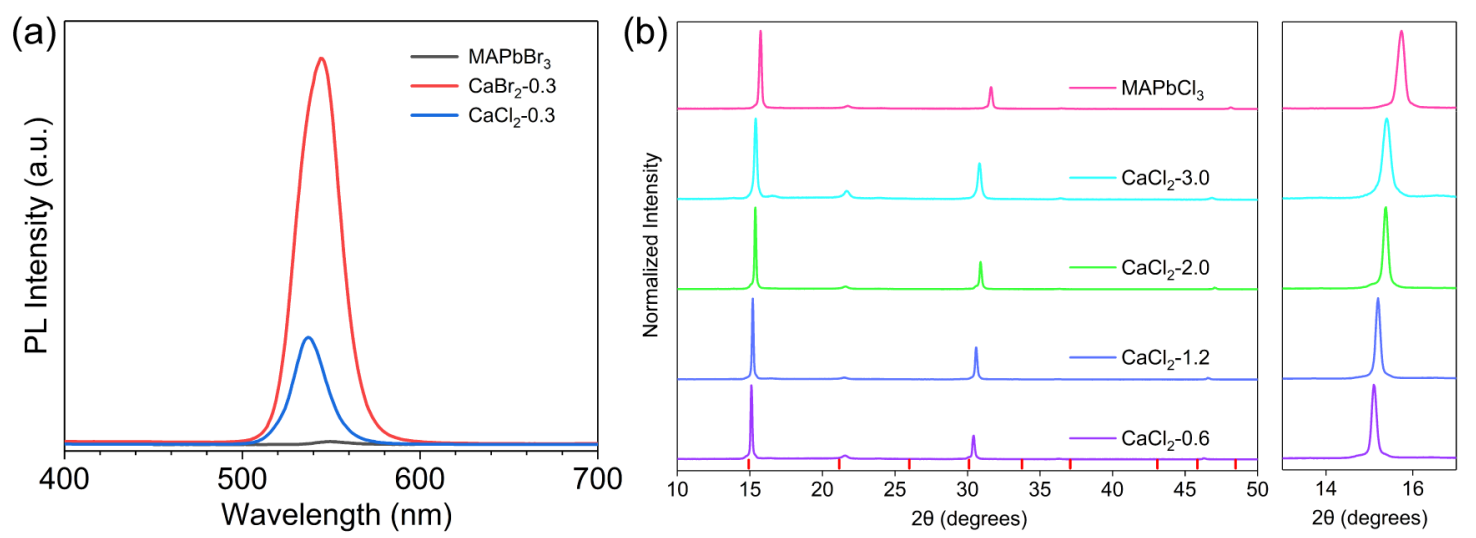

Figure S13. (a) Comparison of the steady-state PL spectra of the $\mathrm{MAPbBr}_{3}$ perovskite films with $\mathrm{CaBr}_{2}$ and $\mathrm{CaCl}_{2}$. (b) The X-ray diffraction patterns of $\mathrm{CaCl}_{2}-0.6, \mathrm{CaCl}_{2}-1.2$, $\mathrm{CaCl}_{2}-2.0, \mathrm{CaCl}_{2}-3.0$ and $\mathrm{MAPbCl}_{3}$ thin films. The vertical bar (red) represents the peak positions of an ideal cubic $\mathrm{MAPbBr}_{3}$ perovskite. 


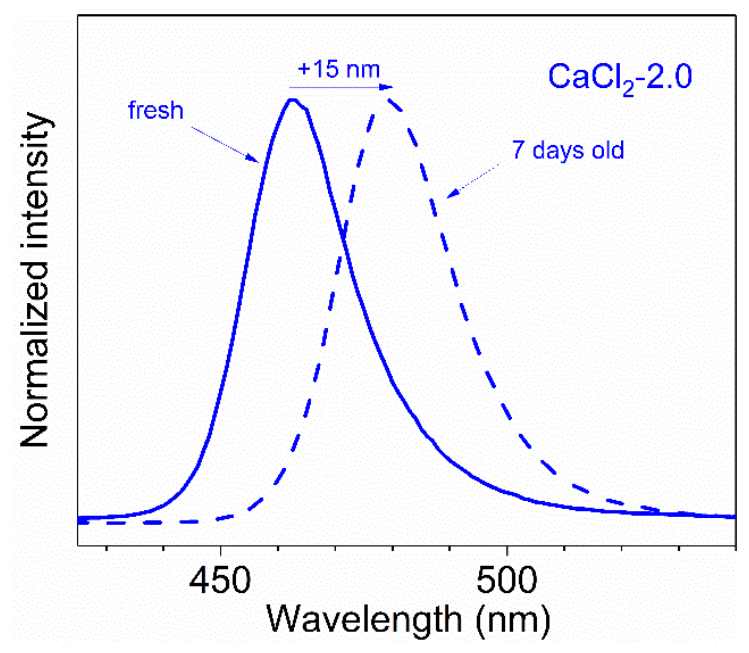

Figure S14. Comparison of PL spectra for the freshly fabricated $\mathrm{CaCl}_{2}-2.0$ thin films (solid line) and 7-day-old thin films (dashed line).
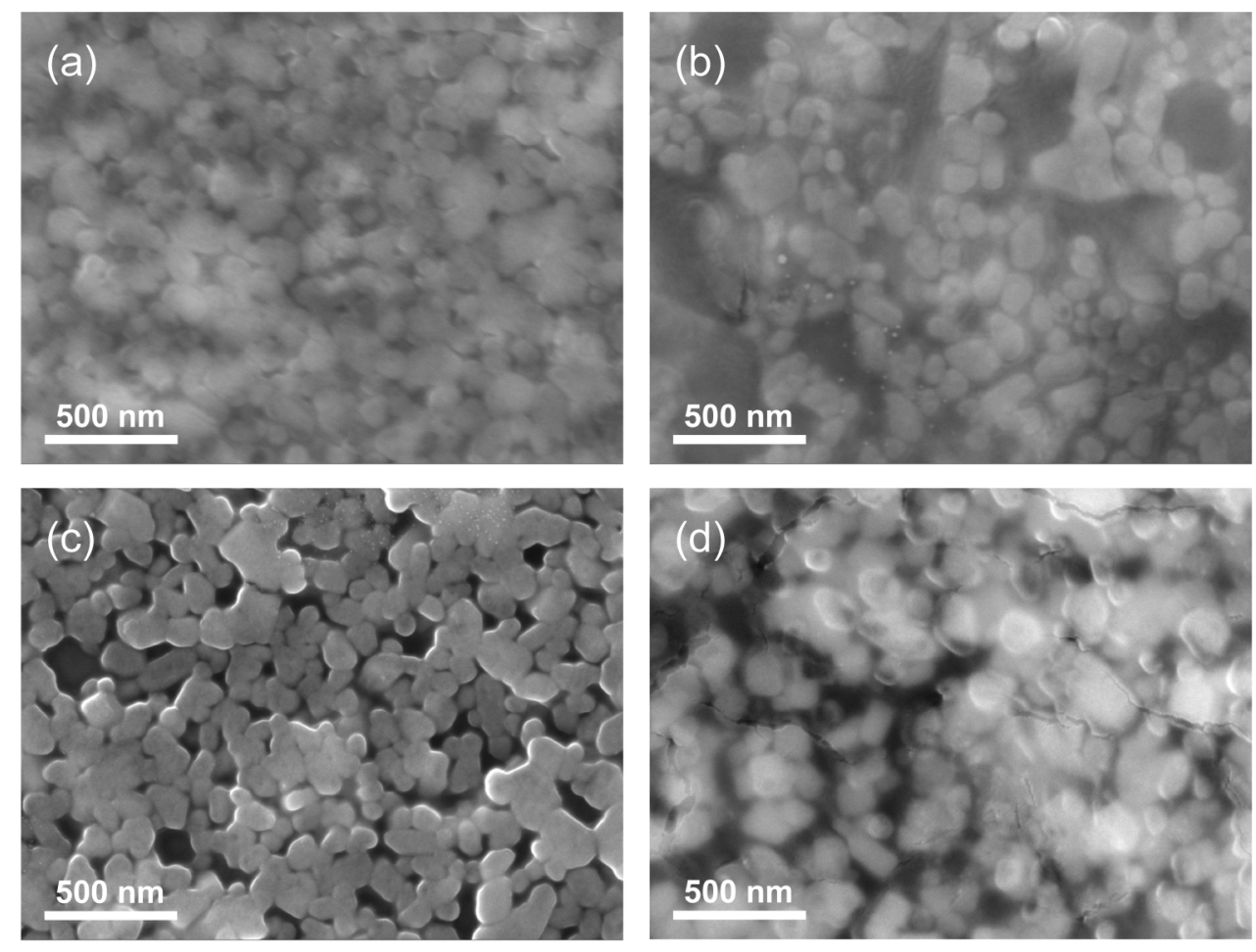

Figure S15. Scanning electron microscopic images of (a) $\mathrm{CaCl}_{2}-0.6$, (b) $\mathrm{CaCl}_{2}-1.2$, (c) $\mathrm{CaCl}_{2}-2.0$ and (d) $\mathrm{CaCl}_{2}-3.0$ thin films 
Table S1. The calculated average crystal size of the perovskite nanocrystals for $\mathrm{MAPbBr}_{3}$, $\mathrm{CaBr}_{2}-0.15, \mathrm{CaBr}_{2}-0.3$, and $\mathrm{CaBr}_{2}-0.6$ films.

\begin{tabular}{cc}
\hline MAPbBr3 & Average size $(\mathrm{nm})$ \\
\hline CaBr2-0.15 & 55.95 \\
CaBr2-0.3 & 55.94 \\
CaBr2-0.6 & 55.93 \\
\hline
\end{tabular}

Table S2. The calculated exciton binding energy from UV-vis absorption spectra for $\mathrm{MAPbBr}_{3}, \mathrm{CaBr}_{2}-0.15, \mathrm{CaBr}_{2}-0.3$, and $\mathrm{CaBr}_{2}-0.6$ films.

\begin{tabular}{cc}
\hline & Exciton binding energy $(\mathrm{meV})$ \\
\hline MAPbBr3 & 47 \\
CaBr2-0.15 & 53 \\
CaBr2-0.3 & 59 \\
CaBr2-0.6 & 53 \\
\hline
\end{tabular}


Table S3. The PL lifetime for $\mathrm{MAPbBr}_{3}, \mathrm{CaBr}_{2}-0.15, \mathrm{CaBr}_{2}-0.3$, and $\mathrm{CaBr}_{2}-0.6$ films.

\begin{tabular}{cccccc}
\hline & $\tau_{1}(\mathrm{~ns})$ & $f_{1}$ & $\tau_{2}(\mathrm{~ns})$ & $f_{2}$ & $\tau_{\text {avg }}(\mathrm{ns})$ \\
\hline MAPbBr3 & 2 & $82 \%$ & 33 & $18 \%$ & 7 \\
$\mathbf{C a B r 2 - 0 . 1 5}$ & 8 & $21 \%$ & 60 & $79 \%$ & 49 \\
$\mathbf{C a B r 2 - 0 . 3}$ & 9 & $30 \%$ & 86 & $70 \%$ & 63 \\
$\mathbf{C a B r 2 - 0 . 6}$ & 3 & $53 \%$ & 59 & $47 \%$ & 30 \\
\hline
\end{tabular}

Table S4. Lattice parameters of optimized structure models. $\left(\alpha=\beta=\gamma=90^{\circ}\right.$ in all cases $)$.

\begin{tabular}{ccccccc}
\hline Supercell & $Z$ & $a(\AA)$ & $b(\AA)$ & $c(\AA)$ & $V\left(\AA^{3}\right)$ & $V / Z\left(\AA^{3}\right)$ \\
\hline $0 \%$ & 4 & 12.246 & 12.060 & 6.023 & 889.6 & 222.4 \\
$25 \%$ & 4 & 12.203 & 11.938 & 5.967 & 869.3 & 217.3 \\
$50 \%$ & 4 & 12.100 & 11.868 & 5.913 & 849.1 & 212.3 \\
$75 \%$ & 4 & 12.004 & 11.760 & 5.856 & 826.7 & 206.7 \\
$100 \%$ & 4 & 11.938 & 11.650 & 5.795 & 806.0 & 201.5 \\
\hline $\mathrm{CaBr} 2$ & 2 & 6.696 & 6.986 & 4.382 & 205.0 & 102.5 \\
\hline
\end{tabular}


Table S5. Absolute VBM energy levels (in eV) of $\mathrm{MAPb}_{1-x} \mathrm{Ca}_{x} \mathrm{Br}_{3}$ and $\mathrm{CaBr}_{2}$.

\begin{tabular}{|c|c|c|c|c|c|c|}
\hline Supercell & Termination & $E_{\text {slab }}^{\text {vac }}$ & $E_{\text {slab }}^{\text {avg }}$ & $E_{\mathrm{VBM}}$ & $E_{\mathrm{F}}$ & VBM \\
\hline \multirow[b]{2}{*}{$0 \%$} & $\mathrm{MABr}$ & 2.902 & -1.592 & -5.001 & \multirow[b]{2}{*}{-0.507} & \multirow[b]{2}{*}{-5.596} \\
\hline & $\mathrm{PbBr}_{2}$ & 3.574 & -2.110 & -6.191 & & \\
\hline \multirow{2}{*}{$25 \%$} & $\mathrm{MABr}$ & 2.906 & -1.647 & -5.198 & \multirow{2}{*}{-0.645} & \multirow{2}{*}{-5.776} \\
\hline & $\mathrm{Pb}_{0.75} \mathrm{Ca}_{0.25} \mathrm{Br}_{2}$ & 3.554 & -2.154 & -6.353 & & \\
\hline \multirow{2}{*}{$50 \%$} & $\mathrm{MABr}$ & 2.890 & -1.687 & -5.155 & \multirow{2}{*}{-0.578} & \multirow{2}{*}{-5.706} \\
\hline & $\mathrm{Pb}_{0.5} \mathrm{Ca}_{0.5} \mathrm{Br}_{2}$ & 3.511 & -2.167 & -6.256 & & \\
\hline \multirow{2}{*}{$75 \%$} & $\mathrm{MABr}$ & 2.891 & -1.738 & -5.445 & \multirow{2}{*}{-0.816} & \multirow{2}{*}{-5.975} \\
\hline & $\mathrm{Pb}_{0.25} \mathrm{Ca}_{0.75} \mathrm{Br}_{2}$ & 3.505 & -2.184 & -6.505 & & \\
\hline \multirow{2}{*}{$100 \%$} & $\mathrm{MABr}$ & 2.878 & -1.789 & -5.546 & \multirow{2}{*}{-0.879} & \multirow{2}{*}{-6.006} \\
\hline & $\mathrm{CaBr}_{2}$ & 3.437 & -2.150 & -6.466 & & \\
\hline $\mathrm{CaBr}_{2}$ & & 3.734 & -1.964 & -6.057 & -0.360 & -6.057 \\
\hline
\end{tabular}


Table S6. Bandedge parameters of $\mathrm{MAPb}_{1-x} \mathrm{Ca}_{x} \mathrm{Br}_{3}$.

\begin{tabular}{lccccc}
\hline Substitution ratio $x$ & $0 \%$ & $25 \%$ & $50 \%$ & $75 \%$ & $100 \%$ \\
\hline Bandgap $E_{\mathrm{g}}(\mathrm{eV})$ & 1.99 & 2.25 & 2.45 & 3.10 & 4.39 \\
Absolute VBM energy level $(\mathrm{eV})$ & -5.60 & -5.78 & -5.71 & -5.97 & -6.01 \\
Electron effective mass $m_{\mathrm{e}}{ }^{*}\left(m_{0}\right)$ & 0.168 & 0.177 & 0.195 & 0.317 & 0.590 \\
Hole effective mass $m_{\mathrm{h}}{ }^{*}\left(m_{0}\right)$ & 0.411 & 0.337 & 0.352 & 2.785 & 1.711 \\
e-h reduced effective mass $\mu_{0}\left(m_{0}\right)$ & 0.119 & 0.116 & 0.126 & 0.285 & 0.439 \\
Dielectric constant $\varepsilon^{\prime}$ at $E_{\mathrm{g}}$ & 5.23 & 5.01 & 4.53 & 4.09 & 4.43 \\
Exciton binding energy $E_{\mathrm{b}}(\mathrm{meV})$ & 59.3 & 62.8 & 83.3 & 231.9 & 303.5 \\
Exciton energy $E_{\mathrm{x}}=E_{\mathrm{g}}-E_{\mathrm{b}}(\mathrm{eV})$ & 1.93 & 2.19 & 2.37 & 2.87 & 4.09 \\
\hline
\end{tabular}

